\title{
Logística del cambio en Instituciones Educativas con hoja de cálculo
}

\section{Change logistics in Educational Institutions with spreadsheet}

\author{
MUÑOZ-LOPEZ, Temístocles $\dagger^{*}$, ESPERICUETA-MEDINA, Marta Nieves, SÁNCHEZ-RIVERA, \\ Lilia y MORÁN-DELGADO, Gabriela
}

Universidad Autónoma de Coahuila. Blvd. V. Carranza s/n. Col. República Oriente C.P.25280 Saltillo, Coah. México.

ID $1^{\mathrm{er}}$ Autor: Temístocles, Muñoz-Lopez / ORC ID: 0000-0003-4940-5730, Researcher ID Tomson: T-7834-2018, CVU CONACYT ID: 202437

ID $1^{\mathrm{er}}$ Coautor: Marta Nieves, Espericueta-Medina / ORC ID: 0000-0002-4924-4332, Researcher ID Web of Science: T1500-2018, arXiv Author ID: Espericueta2018, CVU CONACYT ID: 372705

ID $2^{\text {do }}$ Coautor: Lilia, Sánchez-Rivera / ORC ID: 0000-0001-9468-2599, Researcher ID Tomson: T-1404-2018, CVU CONACYT ID: 613195

ID $3^{\text {er }}$ Coautor: Gabriela, Morán-Delgado / ORC ID: 000001-8433-4216, Researcher ID: X-2070-2018, arXiv Author ID: GMD13, CVU CONACYT ID: 306238

\section{Resumen}

Los procesos de acreditación y certificación son un imperativo de la cultura evaluativa institucional, tomando sus fundamentos en la metodología de la investigación, con una cuidadosa selección de las variables bajo estudio y las categorías analíticas que la sustentan. Este estudio tiene como objetivos elucidar las categorías en que descansa la evaluación institucional, sus fuentes, y su derivación en atributos a evaluar, proceso previo a la elaboración del diagnóstico FODA. El concentrado de las áreas sujetas a evaluación institucional descriptadas y elucidadas es presentado como el límite del presente trabajo con el objetivo de desarrollar una técnica que permita realizar la evaluación institucional y con ella la planeación estratégica en las escuelas o instituciones de educación superior para alcanzar la calidad de los procesos de mejora continua, utilizando en ambos casos (diagnóstico y planeación) una hoja de cálculo. La técnica se probó satisfactoriamente en la autoevaluación de un Programa de Maestría en Ciencias de la Educación de la Universidad Autónoma de Coahuila para integrarlo a la autoevaluación de la información solicitada por los organismos acreditadores. Esta técnica es útil para las instituciones educativas que no tienen programas sofisticados para lograr cambiar y adaptarse a las nuevas realidades educativas.

FODA, Evaluación institucional, Planeación estratégica

\begin{abstract}
The accreditation and certification processes are an imperative of the institutional evaluative culture, taking its foundations in the research methodology, with a careful selection of the variables under study and the analytical categories that support it. This study aims to elucidate the categories in which the institutional evaluation rests, its sources, and its derivation in attributes to be evaluated, a process prior to the elaboration of the SWOT diagnosis. The concentrate of the areas subject to institutional evaluation described and elucidated is presented as the limit of the present work with the objective of developing a technique that allows to carry out the institutional evaluation and with it the strategic planning in the schools or higher education institutions to reach the quality of continuous improvement processes, using in both cases (diagnosis and planning) a spreadsheet. The technique was successfully tested in the self-evaluation of a Master's Program in Education Sciences of the Autonomous University of Coahuila to integrate it to the self-evaluation of the information requested by the accrediting bodies. This technique is useful for educational institutions that do not have sophisticated programs to change and adapt to new educative realities.
\end{abstract}

Citación: MUÑOZ-LOPEZ, Temístocles, ESPERICUETA-MEDINA, Marta Nieves, SÁNCHEZ-RIVERA, Lilia y MORÁN-DELGADO, Gabriela. Logística del cambio en Instituciones Educativas con hoja de cálculo. Revista de Políticas Universitarias. 2019. 3-7: 17-26.

\footnotetext{
* Correspondencia del Autor (correo electrónico: temistoclesmunoz@uadec.edu.mx,)

$\uparrow$ Investigador contribuyendo como primer autor.
} 


\section{Introducción}

La escuela es un importante vehículo a través del cual un grupo social trasmite su historia y complejo de valores, y por ella se forman las personas y se establecen los vínculos económicos, políticos y culturales que mantienen estable a la sociedad o generan los procesos de cambio. La sociedad instituye a la escuela o a las organizaciones que la forman, condicionando en buena medida los procesos de institucionalización de ella.

La evaluación institucional es así un elemento indispensable para mantener la convergencia entre la sociedad y las instituciones educativas. Tendremos que reconocer que pasamos de la era de la planeación a la era de la evaluación, cada vez es más patente la presencia de la acreditación de los programas, la certificación de las instituciones y los alumnos, y los criterios de desempeño de competencias específicas. La evaluación para certificar la calidad utiliza una metodología científica, requiere de indicadores y parámetros, se constata contra referentes específicos o estándares fijados previamente, y reconocidos como legítimos y suficientes (Muñoz López, 2005). En este estudio la evaluación (e-valuatio) es un proceso sistemático, permanente, cíclico y continuo, que permite valorar los programas de trabajo, sus avances, logros, errores y desviaciones, para conocer, corregir y conducir eficientemente los procesos hacia el logro de los objetivos y metas propuestos.

Básicamente, el proceso de evaluación para la acreditación comprende las etapas de autoevaluación o auto-estudios, la evaluación externa y el informe final, que plantea las bases y los tratamientos necesarios para definir o corregir rumbos de calidad y buscar un diagnóstico, a partir de cuyos datos se puedan elaborar los escenarios de su futuro mediato y a largo plazo. Siguiendo con Muñoz López (2005, op. cit.) La autoevaluación conlleva una actitud ética fundamental: Que nunca nuestras palabras sean mejores que nuestros hechos. Para obtener las variables de este estudio se analizan los sistemas educativos desde su perspectiva sistémica, considerando que mantienen una comunicación con factores del entorno (variables) que condicionan o influyen directamente sobre la institución educativa o la carrera en un tiempo y un espacio precisos y con características propias.
Los subsistemas del entorno social que pueden generar amenazas u oportunidades son el político (subsistema de obtención de metas), el económico (subsistema de adaptación) y el cultural (subsistema de integración) por su relación directa con la institución evaluada.

Los elementos internos del sistema educativo se analizan aquí desde tres enfoques para tener una visión integral e integrada y para precisar todos sus elementos constituyentes con un mínimo de tres enfoques a) el enfoque sistémico, b) el estructural y c) el funcional.

Desde el enfoque a) Sistémico el sistema educativo se analiza el subsistema teleológico que comprende los fines de la educación, el subsistema sustantivo. Que comprende el tipo de educación o lo que se enseña, el subsistema metodológico o Normativo que regula cómo se educa y el subsistema topológico que indica donde se da la educación.

El enfoque b) Estructural integra el análisis de la institución desde tres puntos de vista complementarios: como institución, como organización y como comunidad, que convergen simultáneamente para analizarla y facilitar el diagnóstico.

El enfoque c) Funcional se constituye por las tres funciones sustantivas y una adjetiva del sistema educativo. La docencia, la investigación, y la difusión de la cultura. La función adjetiva es la Administración, en tanto que es dependiente de las anteriores y comprende el complejo de elementos infraestructurales que las hace posibles.

Del análisis comprehensivo de la institución, con los tres enfoques se puede fundamentar una evaluación académica o una auditoría institucional. En resumen, podemos afirmar que para evitar omisiones, vacíos, lagunas, inconsistencias y contradicciones, es necesario analizar el sistema educativo sistemáticamente, siguiendo rigurosamente un procedimiento para la obtención de las variables del análisis mediante una destilación o fragmentación de estas categorías. 
Las consideraciones para la evaluación institucional son referidas a los criterios de una metodología científica, con participantes, instrumentos y medios adecuados, para concluir con el logro de los objetivos y propósitos que la iniciaron, sus alcances, y la develación de las debilidades y fortalezas con la construcción de los escenarios a que se enfrentará si cambia o no. Según Chamizo y Jiménez (1982) la Escuela Francesa de Sociología define por primera vez a la sociología como la ciencia de las instituciones, debiéndose a Gurvitch la propuesta de abandonar el concepto y a Rousseau darle un contenido activo (instituir) (Chamizo \& Jiménez S., 1982, págs. 3-12). Las instituciones tienen en su seno una contradicción social entre lo instituido y lo instituyente y cambian en el tiempo generando crisis institucionales que pueden deberse a la divergencia o separación progresiva entre ellas o al desajuste de los elementos que las constituyen.

La Evaluación es el acto de juzgar, es un proceso que concluye en un juicio de valor. Puede decirse que evaluar es el arte de asignar adjetivos en forma fundamentada (Gago Huguet \& Mexico. Secretaría de Educación Pública, 2002). Continuando con Gago Huguet (op. cit.) Evaluar es la base de certificar y acreditar. La evaluación es más una tecnología que una ciencia, su acento está en generar un producto o evaluar el logro de una misión. Es necesario elucidar la situación institucional dado que "Nuestras valuaciones determinan nuestra aproximación a un problema, la definición a nuestros conceptos, la elección de modelos, la selección de nuestras observaciones y la presentación de nuestras conclusiones" (Myrdal, 1978).

Comúnmente se ha aplicado el Análisis FODA como una metodología de estudio de la situación competitiva de una organización en lo externo e interno, considerando sus Fortalezas, Oportunidades, Debilidades y Amenazas. La situación interna se compone de elementos controlables: fortalezas y debilidades, mientras que la situación externa se compone de factores no controlables: oportunidades y amenazas (Orlich., 2008). Uno de los aspectos fundamentales de la planeación estratégica lo constituye el diseño e implantación de estrategias, y así se favorece el desarrollo y ejecución de la planeación formal que facilita la toma de decisiones y el desarrollo de estrategias (Ramírez, 2009).
El proceso de análisis y la elaboración del diagnóstico de los sistemas educativos tiene 2 ámbitos con perspectivas analíticas (categorías) que son, en forma conjunta, su foco de atención (Muñoz López, 2009): a) El análisis del entorno social en que se encuentra inmerso el sistema educativo $\mathrm{y}, \mathrm{b}$ ) El análisis de sus propios componentes internos. Las variables del entorno social se denominan convencionalmente Factores del entorno, en tanto que las variables de la institución se conocen como Elementos del sistema educativo.

El valor agregado de la técnica reside en que se hace rápidamente, no requiere software especial y se pueden modificar directamente sus valores para simular otros resultados.

La hipótesis central se orienta bajo la premisa de que la mejor planeación (institucional $\mathrm{u}$ organizacional) es la que reside en la calidad de tres procesos: Diagnóstico, Pronóstico y aplicación del Tratamiento.

Finalmente podemos considerar esto sirve como una metodología de enseñanza aplicando enfoques de aprender-haciendo y aprendizaje en servicio basada en la caracterización y mejoramiento de procesos logísticos para la formación logística de administradores de empresas que ya ha sido planteado por otros autores (Cano, J. A., \& Ayala, C. J. 2019).

\section{Metodología a desarrollar}

De acuerdo a lo anterior, el objetivo del estudio se orienta a desarrollar una técnica que permita realizar la evaluación institucional y con ella la planeación estratégica, para integrar en las escuelas o instituciones de educación superior la calidad en los procesos de mejora continua mediante procesamiento de datos sin necesidad de alta tecnología, y solo con una hoja de cálculo. En aspectos Metodológicos se siguieron 5 procedimientos descritos en las Actividades:

Se tomaron 13 casos, estudiantes del último semestre de una Maestría en Ciencias de la Educación de universidad pública con el propósito de evaluarla previo al proceso de acreditación del Programa.

\section{Actividad 1. Obtención de variables del estudio}


Se incluyeron 22 variables signalécticas de Información General de la Institución mediante un formato en Word para la evaluación institucional inmediata por juicio de experto, en escala de 0 al 10 en cada una de las 87 variables o elementos internos de la institución y los 40 factores del entorno considerando solo las 33 variables pertinentes de acuerdo a Yarce, Jorge (2010. Megatendencias de la Educación).

Las variables signalécticas fueron:

1. Nombre de la Institución.

2. Nombre del Evaluador.

3. Fecha.

4. Clave C. Trabajo.

5. Nombre CT.

6. Turno.

7. Zona Escolar.

8. Jefatura de Sector.

9. Dep. Administrativa.

10. Dep. Normativa.

11. Servicio.

12. Sostenimiento.

13. Estatus.

14. Nombre del Director(a).

15. Municipio.

16. Localidad.

17. Región.

18. Subregión.

19. Domicilio.

20. Colonia.

21. Teléfono.

22. Código Postal.
Los componentes o variables analizadas (organizadas en renglones de la base de datos) se derivan del análisis de a) El Subsistema teleológico o los fines de la educación, b) el Subsistema sustantivo que dicta lo que se enseña y aprende, el c) Subsistema normativo o de las regulaciones formales y el d) Subsistema topológico dónde se da la educación a los cuales se agregó la valoración del cumplimiento de los Reguladores jurídico-sociales. Por otra parte, las Perspectivas o visiones posibles de la institución incluyen las categorías (columnas en la hoja de cálculo) de: a) la Docencia, b) la Comunidad, c) la Organización, c) la Administración y d) la Institución.

Cada elemento obtenido fue valorado primero en una hoja de procesador de textos considerando la Existencia de la variable, la Penetración en la comunidad correspondiente, la Calidad observada en la variable y, si está incluida al proceso de Mejora continua. Cada nivel aporta 2.5 puntos a la evaluación de cada elemento, lo que da un total de 10 puntos. De la destilación, separación o rompimiento de estas categorías se obtuvieron 87 elementos o variables a medir durante la evaluación.

Además, se analizaron fuera esta escala de 0-10, los Indicadores escolares de eficiencia en una tabulación donde se comparan a nivel Institución, Ciudad, Estatal y Nacional por columnas y 11 indicadores de: Retención, Deserción, Repetición, Reprobación, Aprobación, Eficiencia terminal, Relación No. Estudiantes por escuela, Relación No. Estudiantes por grupo, Relación No. de Estudiantes por maestro, Relación Número de grupos por escuela, y finalmente la Relación Número de maestros por escuela. Finalmente se analizan históricamente los indicadores de la serie de los últimos 5 años en los indicadores de Población de Estudiantes, Número de Maestros, Número de Egresados, Número de Aulas, Número de Laboratorios, Número de Libros, Número de Computadoras, Metros cuadrados de Áreas de Recreación, Número Programas sociales, Número Personal de Confianza, y Número Personal Sindicalizado. Con el formato para evaluación institucional inmediata en Word, y utilizando el criterio de juicio de experto se elaboraron instrumentos para concentrar la información en escala de 0 al 10 en cada una de las variables del estudio, mediante la suma de cuatro opciones progresivas en cada variable con valor de $0-2.5$, para tener el 10 , como el puntaje total.

MUÑOZ-LOPEZ, Temístocles, ESPERICUETA-MEDINA, Marta Nieves, SÁNCHEZ-RIVERA, Lilia y MORÁN-DELGADO, Gabriela. Logística del cambio en Instituciones Educativas con hoja de cálculo. Revista de Políticas Universitarias. 2019 


\begin{tabular}{|c|c|}
\hline \multicolumn{2}{|c|}{$\begin{array}{l}\text { 1. SUBSISTEMA Teleológico: Los fines de la } \\
\text { educación }\end{array}$} \\
\hline \multirow[t]{3}{*}{ La Docencia } & $\begin{array}{l}1 \text { Los maestros conocen la Misión } \\
\text { de la carrera }\end{array}$ \\
\hline & $\begin{array}{l}2 \text { Los maestros conocen la } \\
\text { Filosofía en la carrera }\end{array}$ \\
\hline & $\begin{array}{l}3 \text { Los maestros conocen los } \\
\text { Propósitos de la carrera }\end{array}$ \\
\hline \multirow[t]{3}{*}{ La Comunidad } & $\begin{array}{l}4 \text { Los maestros y alumnos } \\
\text { conocen y practican los Valores } \\
\text { institucionales en la carrera }\end{array}$ \\
\hline & $\begin{array}{l}5 \text { Los maestros y alumnos tienen } \\
\text { calidad en sus Valores personales }\end{array}$ \\
\hline & $\begin{array}{l}6 \text { Los maestros y alumnos } \\
\text { respetan las Normas de relación } \\
\text { en la carrera }\end{array}$ \\
\hline \multirow[t]{3}{*}{ La Organización } & $\begin{array}{l}7 \text { El Programa tiene Planes de } \\
\text { futuro muy claros }\end{array}$ \\
\hline & $\begin{array}{l}8 \text { El proceso académico tiene } \\
\text { Programas bien elaborados }\end{array}$ \\
\hline & $\begin{array}{l}9 \text { Hay Estrategias de desarrollo a } \\
\text { corto, mediano y largo plazo }\end{array}$ \\
\hline \multirow[t]{4}{*}{ La Administración } & $\begin{array}{l}10 \text { La Administración del } \\
\text { Programa se hace con Valores }\end{array}$ \\
\hline & $\begin{array}{l}11 \text { La Administración del } \\
\text { Programa se hace con Principios } \\
\text { Humanos }\end{array}$ \\
\hline & $\begin{array}{l}12 \text { La Administración del } \\
\text { Programa se hace con Políticas } \\
\text { claras }\end{array}$ \\
\hline & $\begin{array}{l}13 \mathrm{La} \text { Administración del } \\
\text { Programa tiene Prioridades }\end{array}$ \\
\hline \multirow[t]{3}{*}{ La Institución } & $\begin{array}{l}\text { 14 La escuela administra el } \\
\text { Programa con Visión }\end{array}$ \\
\hline & $\begin{array}{l}15 \text { La escuela administra el } \\
\text { Programa con un Proyecto claro }\end{array}$ \\
\hline & $\begin{array}{l}16 \text { La escuela administra el } \\
\text { Programa con Principios de } \\
\text { Calidad }\end{array}$ \\
\hline \multicolumn{2}{|c|}{ 2. SUBSISTEMA Sustantivo: Lo que se enseña y aprende } \\
\hline \multirow{10}{*}{$\begin{array}{l}\text { El perfil de la } \\
\text { estructura Didáctico } \\
\text { Matética. Elementos } \\
\text { del Acto educativo }\end{array}$} & $\begin{array}{l}17 \text { Los maestros: su Capacidad } \\
\text { didáctica, experiencia profesional }\end{array}$ \\
\hline & $\begin{array}{l}18 \text { Los objetivos de los cursos } \\
\text { tienen Claridad y se cumplen }\end{array}$ \\
\hline & $\begin{array}{l}19 \text { Las habilidades de los maestros } \\
\text { para educar tienen Diversidad y } \\
\text { creatividad }\end{array}$ \\
\hline & $\begin{array}{l}20 \text { Los estudiantes en los cursos } \\
\text { tienen Responsabilidad, } \\
\text { participación y disciplina grupal }\end{array}$ \\
\hline & $\begin{array}{l}21 \text { Los contenidos de los cursos } \\
\text { son de Calidad, interesantes y con } \\
\text { utilidad }\end{array}$ \\
\hline & $\begin{array}{l}22 \text { La Relación profesor - alumno } \\
\text { es de Amistad y cordialidad }\end{array}$ \\
\hline & $\begin{array}{l}23 \text { La enseñanza tiene materiales } \\
\text { de calidad, métodos y técnicas } \\
\text { adecuados }\end{array}$ \\
\hline & $\begin{array}{l}24 \text { La Programación de los temas } \\
\text { es de Orden, secuencial y con } \\
\text { organización }\end{array}$ \\
\hline & $\begin{array}{l}25 \text { El Aprendizaje promueve la } \\
\text { Proactividad, autonomía, novedad } \\
\text { y aplicaciones }\end{array}$ \\
\hline & $\begin{array}{l}26 \text { El Ambiente físico tiene } \\
\text { comodidad, seguridad y } \\
\text { adecuación }\end{array}$ \\
\hline
\end{tabular}

\begin{tabular}{|c|c|}
\hline \multirow[t]{5}{*}{$\begin{array}{l}\text { La Comunidad en el } \\
\text { aprendizaje }\end{array}$} & $\begin{array}{l}27 \text { Se desarrollan en los } \\
\text { estudiantes los Conocimientos } \\
\text { más actualizados }\end{array}$ \\
\hline & $\begin{array}{l}28 \text { Se desarrollan en los } \\
\text { estudiantes los mejores Valores } \\
\text { morales y éticos necesarios en el } \\
\text { mundo del trabajo }\end{array}$ \\
\hline & $\begin{array}{l}29 \text { Se desarrollan en los } \\
\text { estudiantes los Hábitos } \\
\text { demandados por la profesión }\end{array}$ \\
\hline & $\begin{array}{l}30 \text { Se desarrollan en los } \\
\text { estudiantes las mejores Actitudes } \\
\text { para apoyar su convivencia social }\end{array}$ \\
\hline & $\begin{array}{l}31 \text { Se desarrollan en los } \\
\text { estudiantes las Aptitudes } \\
\text { necesarias para su competencia en } \\
\text { el mundo del trabajo }\end{array}$ \\
\hline \multirow[t]{3}{*}{$\begin{array}{l}\text { La Organización } \\
\text { académica }\end{array}$} & $\begin{array}{l}32 \text { Los Cuerpos académicos son } \\
\text { de calidad y promueven la } \\
\text { superación de sus miembros y su } \\
\text { colegiado }\end{array}$ \\
\hline & $\begin{array}{l}33 \text { La Información académica de } \\
\text { los cursos está actualizada con el } \\
\text { apoyo de la administración }\end{array}$ \\
\hline & $\begin{array}{l}34 \text { La Infraestructura de apoyo } \\
\text { docente facilita a la organización } \\
\text { el apoyo académico de los } \\
\text { docentes }\end{array}$ \\
\hline \multirow[t]{4}{*}{$\begin{array}{l}\text { La Administración } \\
\text { Académica }\end{array}$} & $\begin{array}{l}\text { 35 Se mantiene un excelente } \\
\text { Control escolar histórico para } \\
\text { analizar las tendencias de la } \\
\text { matrícula y las calificaciones }\end{array}$ \\
\hline & $\begin{array}{l}36 \text { La Infraestructura de apoyo al } \\
\text { control académico está actualizada } \\
\text { y es de calidad }\end{array}$ \\
\hline & $\begin{array}{l}37 \text { Se mantiene un Control } \\
\text { Docente tolerante y respetuoso }\end{array}$ \\
\hline & $\begin{array}{l}38 \text { Se mantiene un excelente } \\
\text { Control de Kardex }\end{array}$ \\
\hline \multirow[t]{4}{*}{$\begin{array}{l}\text { La Institución en la } \\
\text { comunidad }\end{array}$} & $\begin{array}{l}39 \text { El Prestigio institucional en la } \\
\text { comunidad es alto }\end{array}$ \\
\hline & $\begin{array}{l}40 \text { La institución tiene un } \\
\text { Posicionamiento firme en la } \\
\text { comunidad }\end{array}$ \\
\hline & $\begin{array}{l}41 \text { La Imagen institucional es } \\
\text { apreciada en la comunidad }\end{array}$ \\
\hline & $\begin{array}{l}42 \text { Los Egresados son apreciados } \\
\text { laboralmente y gozan de prestigio }\end{array}$ \\
\hline \multicolumn{2}{|c|}{ 3. SUBSISTEMA Normativo: Regulaciones formales } \\
\hline \multirow[t]{5}{*}{$\begin{array}{l}\text { La Administración de } \\
\text { la Docencia }\end{array}$} & $\begin{array}{l}43 \text { Los Calendarios se presentan y } \\
\text { adecuan con oportunidad para } \\
\text { orientar las labores académicas }\end{array}$ \\
\hline & $\begin{array}{l}44 \text { Existen y se respetan los } \\
\text { Horarios y compromisos } \\
\text { académicos }\end{array}$ \\
\hline & $\begin{array}{l}45 \text { Hay Registros Académicos de } \\
\text { cada uno de los docentes y } \\
\text { valoraciones de su desempeño }\end{array}$ \\
\hline & $\begin{array}{l}46 \text { Existen y se aplican los } \\
\text { Reglamentos para regular las } \\
\text { labores de los docentes }\end{array}$ \\
\hline & $\begin{array}{l}47 \text { Existen y se aplican Perfiles } \\
\text { definidos para el ingreso, } \\
\text { permanencia y desarrollo } \\
\text { académico de los docentes }\end{array}$ \\
\hline
\end{tabular}




\begin{tabular}{|c|c|}
\hline \multirow[t]{3}{*}{$\begin{array}{l}\text { Las normas de la } \\
\text { Comunidad }\end{array}$} & $\begin{array}{l}48 \text { Existen Reglamentos que } \\
\text { regulan las relaciones entre los } \\
\text { miembros de la comunidad }\end{array}$ \\
\hline & $\begin{array}{l}\text { 49 Las normas se orientan a crear, } \\
\text { desarrollar y mantener } \\
\text { Asociaciones que apoyen los } \\
\text { Programas académicos }\end{array}$ \\
\hline & $\begin{array}{l}50 \text { Hay regulaciones para el } \\
\text { comportamiento de la comunidad } \\
\text { en sus actividades Extramuros }\end{array}$ \\
\hline \multirow{2}{*}{$\begin{array}{l}\text { La estructura y } \\
\text { funciones de la } \\
\text { Organización }\end{array}$} & $\begin{array}{l}51 \text { Existen y se aplican los } \\
\text { Manuales de la Organización }\end{array}$ \\
\hline & $\begin{array}{l}52 \text { Se aplican atenciones } \\
\text { especiales de la administración } \\
\text { para apoyar a los Docentes }\end{array}$ \\
\hline \multirow[t]{3}{*}{$\begin{array}{l}\text { La normatividad de la } \\
\text { Administración }\end{array}$} & $\begin{array}{l}53 \text { Existe y se aplica el Perfil de } \\
\text { los Administradores }\end{array}$ \\
\hline & $\begin{array}{l}54 \text { El mantenimiento y uso de la } \\
\text { infraestructura está regulado y } \\
\text { normado }\end{array}$ \\
\hline & $\begin{array}{l}55 \text { Existen y se aplican los } \\
\text { Manuales de procedimientos }\end{array}$ \\
\hline \multirow[t]{3}{*}{$\begin{array}{l}\text { La Institución en el } \\
\text { entorno social }\end{array}$} & $\begin{array}{l}56 \text { La institución es considerada } \\
\text { como una organización Ética, al } \\
\text { igual que todos sus programas } \\
\text { educativos }\end{array}$ \\
\hline & $\begin{array}{l}57 \text { Benefactores de la sociedad a } \\
\text { la carrera }\end{array}$ \\
\hline & $\begin{array}{l}58 \text { Servicio social voluntario y/o } \\
\text { obligatorio }\end{array}$ \\
\hline \multicolumn{2}{|c|}{ 4. SUBSISTEMA Topológico: dónde se da la educación } \\
\hline \multirow[t]{4}{*}{$\begin{array}{l}\text { El espacio vital de la } \\
\text { Docencia }\end{array}$} & $\begin{array}{l}59 \text { Las Aulas tienen la comodidad } \\
\text { e infraestructura adecuadas para } \\
\text { las sesiones de trabajo }\end{array}$ \\
\hline & $\begin{array}{l}60 \text { Las Prácticas están integradas } \\
\text { como son parte del modo de vida } \\
\text { cotidiano del docente }\end{array}$ \\
\hline & $\begin{array}{l}\text { 61 La Biblioteca y los recursos } \\
\text { bibliográficos digitales e impresos } \\
\text { apoyan con suficiencia a los } \\
\text { docentes }\end{array}$ \\
\hline & $\begin{array}{l}62 \text { La Informática es de alta } \\
\text { calidad y se mantiene como parte } \\
\text { de la vida cotidiana de la docencia }\end{array}$ \\
\hline \multirow[t]{3}{*}{$\begin{array}{l}\text { El espacio vital de la } \\
\text { Comunidad }\end{array}$} & $\begin{array}{l}63 \text { Los Espacios para la movilidad } \\
\text { de la comunidad son suficientes, } \\
\text { adecuados y seguros }\end{array}$ \\
\hline & $\begin{array}{l}\text { 64 La Infraestructura para la vida } \\
\text { cotidiana de la comunidad es } \\
\text { suficiente, adecuada y segura }\end{array}$ \\
\hline & $\begin{array}{l}65 \text { Los Accesos para la movilidad } \\
\text { cotidiana son suficientes, } \\
\text { adecuados y seguros }\end{array}$ \\
\hline \multirow[t]{3}{*}{$\begin{array}{l}\text { El espacio vital de la } \\
\text { Organización }\end{array}$} & $\begin{array}{l}66 \text { Los Espacios asignados a los } \\
\text { departamentos son suficientes, } \\
\text { adecuados y seguros }\end{array}$ \\
\hline & $\begin{array}{l}67 \text { La Infraestructura es adecuada } \\
\text { y segura }\end{array}$ \\
\hline & $\begin{array}{l}68 \text { Los Accesos son adecuados y } \\
\text { seguros }\end{array}$ \\
\hline \multirow[t]{3}{*}{$\begin{array}{l}\text { El espacio vital de la } \\
\text { Administración }\end{array}$} & $\begin{array}{l}69 \text { Espacio Administrativo } \\
\text { adecuado del Consejo de } \\
\text { Administración o equivalente }\end{array}$ \\
\hline & $\begin{array}{l}70 \text { Infraestructura para el } \\
\text { desarrollo de actividades de los } \\
\text { Directivos }\end{array}$ \\
\hline & $\begin{array}{l}71 \text { Espacios Infraestructura de } \\
\text { calidad para la administración }\end{array}$ \\
\hline
\end{tabular}

\begin{tabular}{|c|c|}
\hline \multirow{2}{*}{$\begin{array}{l}\text { El espacio vital de la } \\
\text { Institución }\end{array}$} & 72 Ubicación geográfica \\
\hline & $\begin{array}{l}73 \text { Área de influencia de la } \\
\text { matrícula }\end{array}$ \\
\hline \multicolumn{2}{|c|}{ 5. Reguladores Jurídico Sociales } \\
\hline \multirow{3}{*}{$\begin{array}{l}\text { La acreditación y } \\
\text { certificación de la } \\
\text { Docencia }\end{array}$} & $\begin{array}{l}74 \text { Normas que obligan a } \\
\text { certificarse }\end{array}$ \\
\hline & 75 Infraestructura para la docencia \\
\hline & $\begin{array}{l}76 \text { Gestión académica de los } \\
\text { docentes }\end{array}$ \\
\hline \multirow[t]{3}{*}{$\begin{array}{l}\text { La acreditación y } \\
\text { certificación de la } \\
\text { Comunidad }\end{array}$} & $\begin{array}{l}77 \text { Organizaciones acreditadoras } \\
\text { y/o certificadoras de grupos } \\
\text { internos }\end{array}$ \\
\hline & $\begin{array}{l}78 \text { Benefactores acreditados } \\
\text { apoyan el Programa }\end{array}$ \\
\hline & $\begin{array}{l}79 \text { Asociaciones de la comunidad } \\
\text { acreditan el trabajo de los } \\
\text { programas }\end{array}$ \\
\hline \multirow{4}{*}{$\begin{array}{l}\text { La acreditación y } \\
\text { certificación de la } \\
\text { Organización }\end{array}$} & $\begin{array}{l}80 \text { RVOE de la carrera en toda su } \\
\text { totalidad de opciones }\end{array}$ \\
\hline & $\begin{array}{l}81 \text { Acreditación de las } \\
\text { competencias directivas }\end{array}$ \\
\hline & $\begin{array}{l}82 \text { Infraestructura de la } \\
\text { organización }\end{array}$ \\
\hline & $\begin{array}{l}83 \text { Programas para lograr la } \\
\text { acreditación y certificación en } \\
\text { todos los ámbitos del Programa }\end{array}$ \\
\hline \multirow{2}{*}{$\begin{array}{l}\text { La acreditación y } \\
\text { certificación de la } \\
\text { Administración }\end{array}$} & $\begin{array}{l}84 \text { La Contabilidad está } \\
\text { certificada en todos sus procesos }\end{array}$ \\
\hline & $\begin{array}{l}85 \text { Se acreditan los Perfiles de los } \\
\text { Docentes y de los Estudiantes }\end{array}$ \\
\hline \multirow[t]{2}{*}{$\begin{array}{l}\text { La acreditación y } \\
\text { certificación de la } \\
\text { Institución }\end{array}$} & $\begin{array}{l}86 \text { Las Relaciones Públicas } \\
\text { acreditan y certifican a la } \\
\text { institución y sus programas }\end{array}$ \\
\hline & $\begin{array}{l}87 \text { Se acreditan y certifican los } \\
\text { protocolos de Gestión de procesos }\end{array}$ \\
\hline
\end{tabular}

Tabla 1 Listado de los Elementos (variables de la institución) considerados en la evaluación del sistema educativo donde se ponderan las variables de la segunda columna, Existencia del atributo (2.5), su Penetración en la comunidad (2.5), su Calidad (2.5), su Mejora continua (2.5), lo que suma (10) cuando se está en total acuerdo en que se cumplen los criterios para cada variable

Adicionalmente se tomaron los 40 factores del entorno considerando solo 33 variables pertinentes de acuerdo a Yarce, Jorge (2010. Megatendencias de la Educación) que se enlistan en la Tabla 2: 


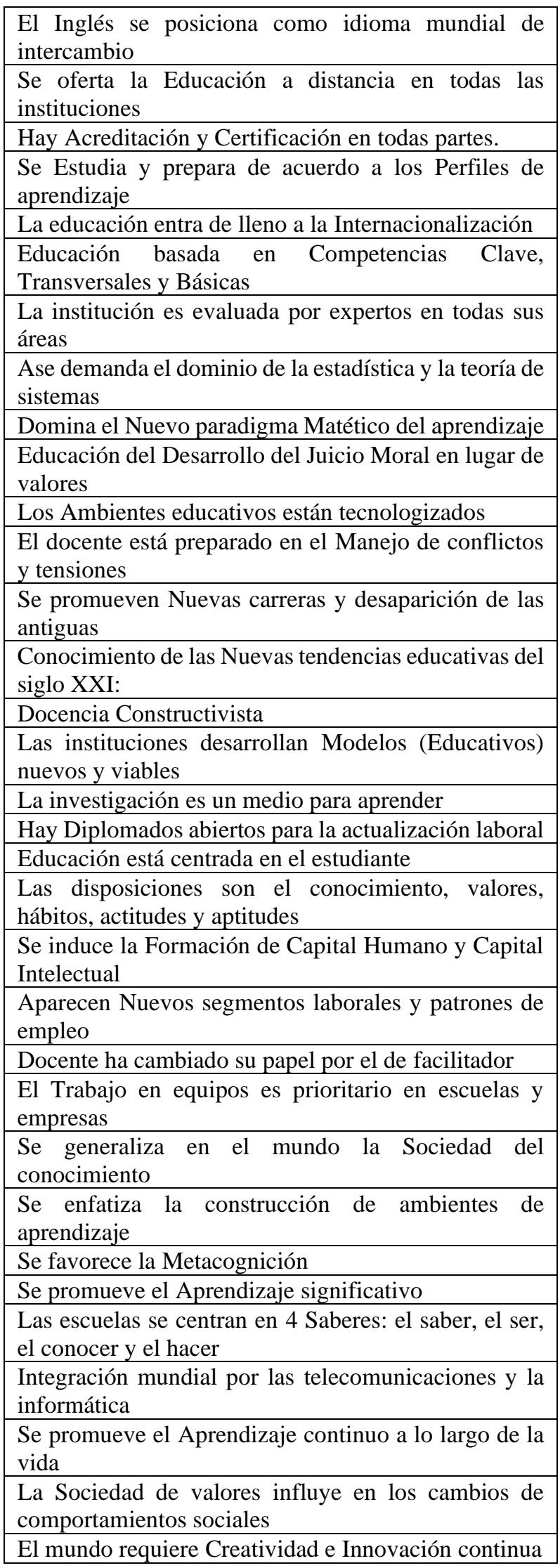

Tabla 2 Tendencias en los factores del Entorno de acuerdo a Jorge Yarce

En nuestro caso se emplea la metodología FODA del IPN (Instituto Politécnico Nacional;, 2002, pág. 7).

\section{Actividad 2. Arreglo de los datos y tratamiento preliminar}

Las variables obtenidas de la evaluación en Word, son ubicadas en la Hoja 1 del archivo de Excel con sus valores en tal como se observan en la ¡Error! No se encuentra el origen de la referencia., que adicionalmente pueden ser clasificados con valores normales, superiores o inferiores utilizando el criterio de la media \pm una desviación estándar.

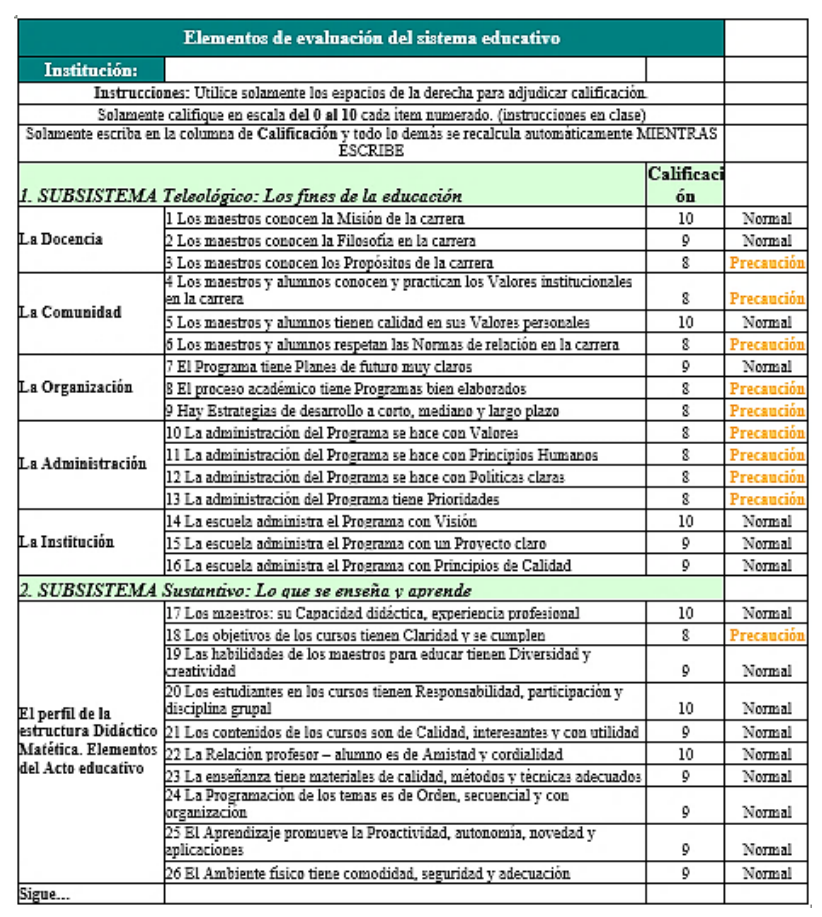

Figura 1 Ubicación de las variables en hoja de cálculo con los valores obtenidos de su ponderación por cada uno de los expertos

Posteriormente los elementos del sistema educativo se copian en la hoja 2 donde se ordenan de acuerdo a sus calificaciones de forma descendente, con lo cual separamos los valores mayores identificados como Fortalezas internas y los menores que serán considerados Debilidades internas.

Lo anterior se observa en la Figura 2 donde las fortalezas son los elementos que tienen los valores mayores y las debilidades las que tienen los valores más bajos. 


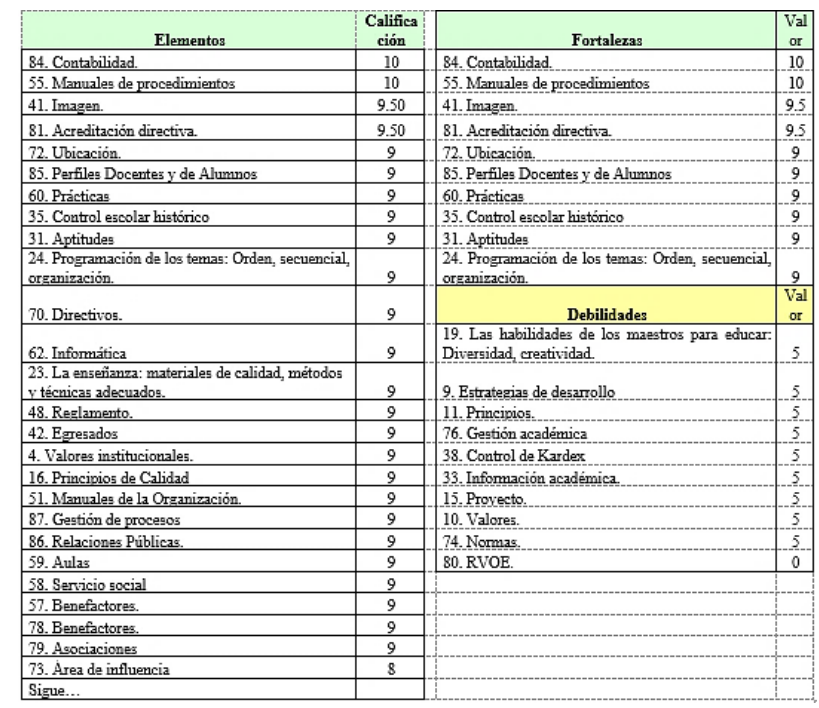

Figura 2 Evaluación de los elementos de la institución de acuerdo al subsistema al que pertenecen, valores descendentes para separar las Fortalezas (valores más altos) y Debilidades (valores más bajos)

Lo mismo ocurre con la lista de factores que se anotan en la hoja 3 y se ordenan de manera descendente para separar las oportunidades y amenazas para seguir el mismo procedimiento que los Elementos de la hoja anterior y obtener las Oportunidades con los valores más altos, y las Amenazas con los más bajos, como se observa en la Figura 3.

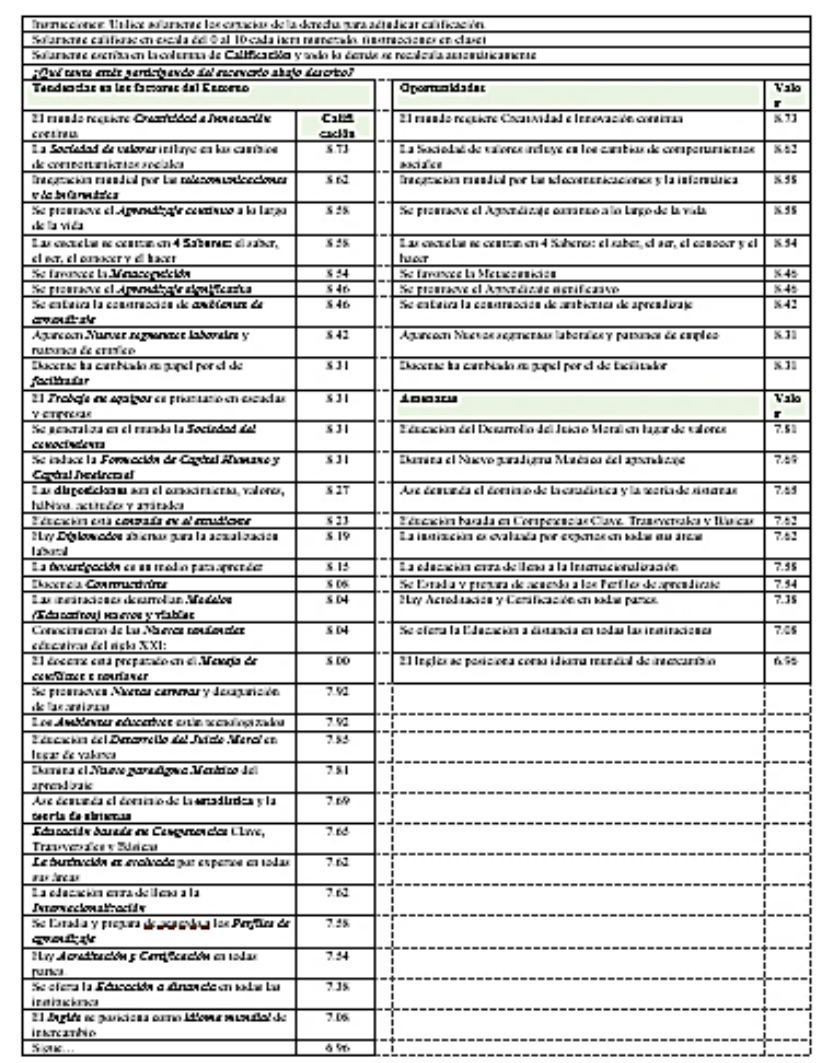

Figura 3 Factores del entorno ordenados según sus valores descendentes para separar las Oportunidades (valores más altos) y Amenazas (valores más bajos)

\section{Actividad 3. Graficando los datos}

El siguiente paso o actividad de contrastación, realizada en hoja aparte (hoja 4) requiere ponderar la información previamente, contrastando los valores obtenidos en términos semejantes a los de probabilidad estadística, asignando valores decimales entre 0 y 1 a cada Debilidad o Potencialidad de los Elementos del sistema, y a cada Oportunidad o Amenaza de los Factores del entorno. Esto ajusta los valores en condiciones más realistas, como se observa en la Figura 4.

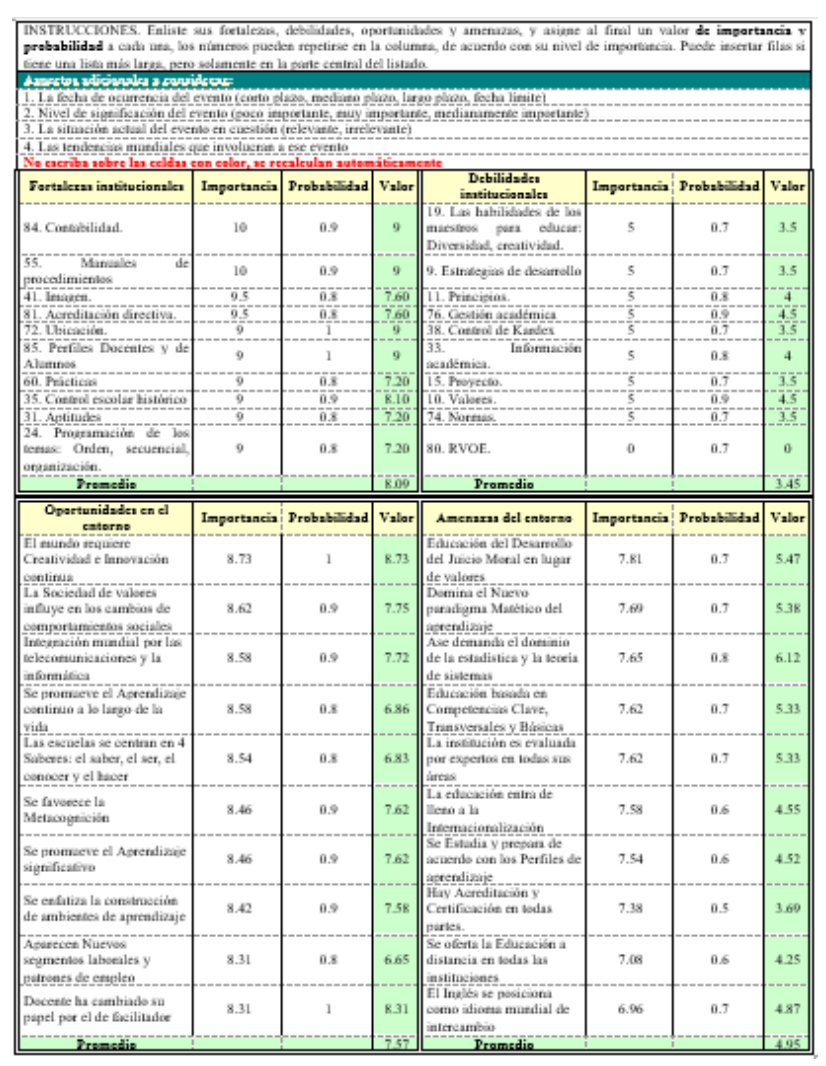

Figura 4 Figura que muestra los atributos evaluados con sus niveles de importancia y su multiplicación que los pondera con la probabilidad de ocurrencia para obtener el valor final

Ahora el siguiente paso es graficar los resultados para hacer una lectura más comprensible, lo que puede hacerse en la misma hoja para graficarse como se observa en la Figura 5, donde ya tenemos la visión completa de la posición competitiva de la institución. 


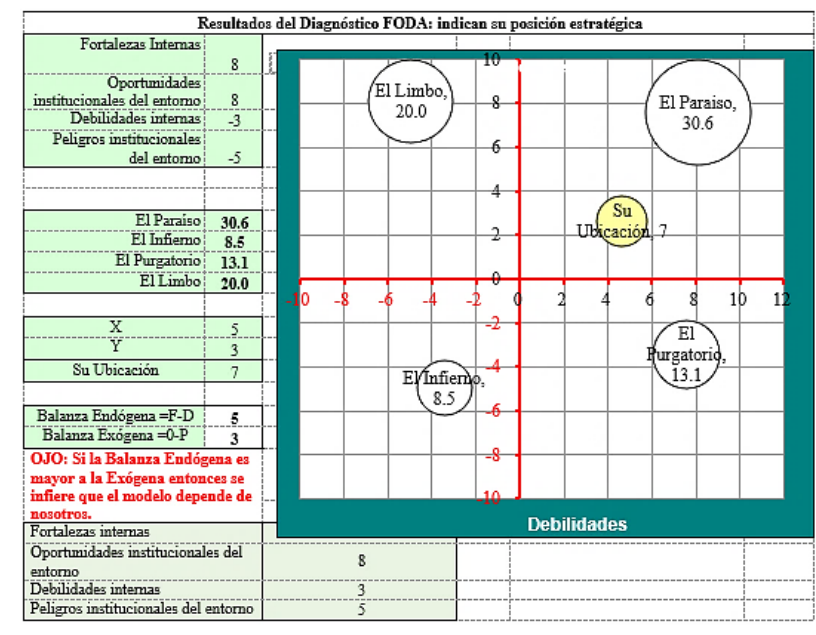

Figura 5 Posición gráfica de la institución que denota las áreas que requieren y hacen posible la intervención para mejorarla

\section{Actividad 4. Organizando la información para la planeación estratégica}

La información se organiza en una hoja aparte haciendo converger tanto Elementos como Factores usando la función "concatenar" para contrastarlos en los cuatro cuadrantes las distintas combinaciones de FODA:

1. FO (Maxi-Maxi). Estrategia para maximizar tanto las Fortalezas como las Oportunidades.

2. DO (Mini-Maxi) Estrategia para minimizar las Debilidades y maximizar las Oportunidades.

3. FA (Maxi-Mini) Estrategia para aprovechar las Fortalezas la Institución y minimizar las Amenazas.

4. DA (Mini-Mini) Estrategia para minimizar tanto las Amenazas como las Debilidades.

Esto se logra con la función antedicha, por ejemplo, para concatenar Fortalezas/Oportunidades se refiere automáticamente las celdas relacionadas $=$ CONCATENAR(FODA!A12,FODA!A25) como se observa en la Figura 6.

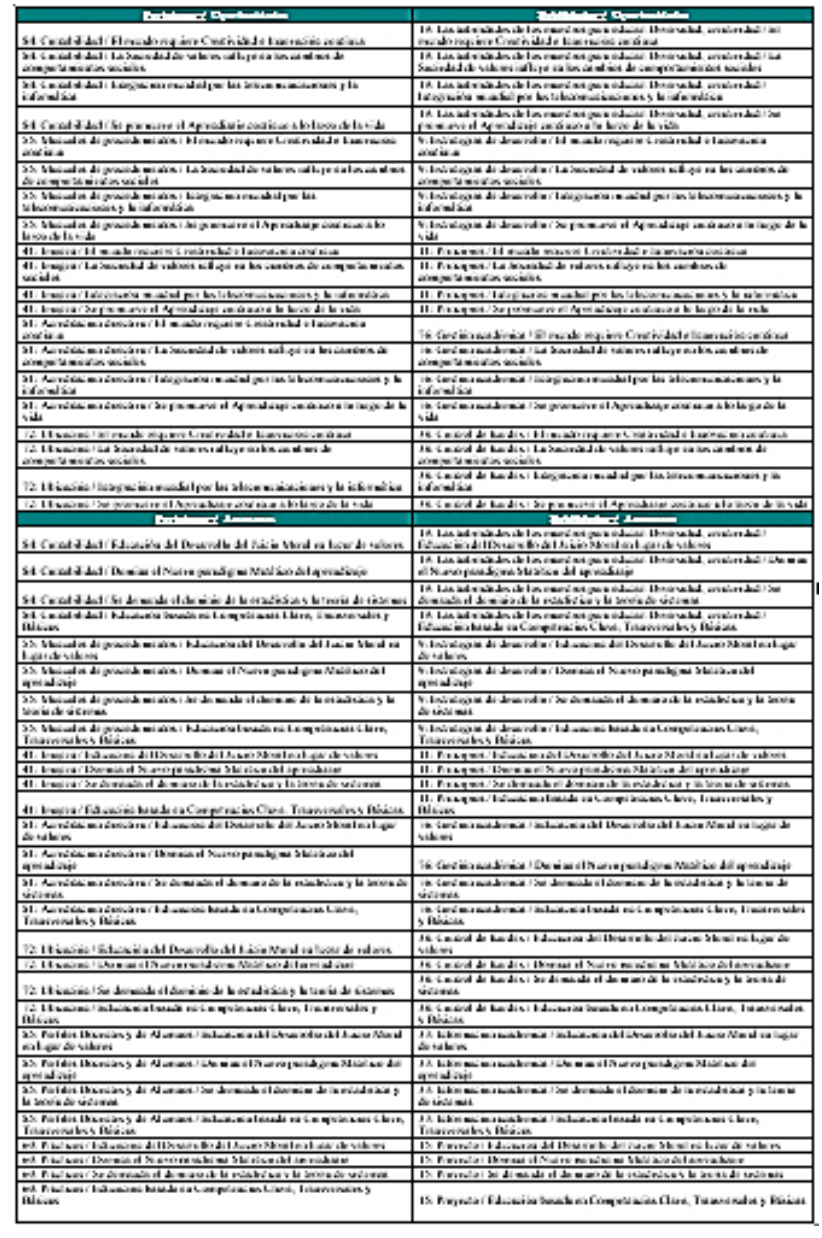

Figura 6 Cuadro que concentra los resultados del diagnóstico para proceder a diseñar las estrategias

\section{Actividad 5. El diseño de estrategias}

La siguiente fase o actividad consiste en redactar las estrategias que aparecen en la figura anterior y jerarquizarlas para aplicarlas o resolverlas en los programas de desarrollo institucional.

\section{Resultados}

La prueba realizada para analizar la técnica se aplicó a un programa educativo que requiere acreditación y sus datos sirvieron como evidencia empírica del uso de la técnica y del diagnóstico institucional.

La metodología descrita y aplicada es confiable y flexible para introducir los conceptos y los valores numéricos para aplicarla. Es más susceptible de modificación el listado de factores del entorno que se sugieran dado que cada institución vive realidades y contextos diferentes, no así el listado de los Elementos que son en gran parte comunes de todas las organizaciones educativas. 
En los resultados graficados y presentados en el estudio completo se observa la situación de la institución y los posibles ámbitos de desarrollo, para analizar cómo se pueden corregir las debilidades y aprovechar las oportunidades. Cada etapa del proceso graficado muestra el resultado de la aplicación de la Técnica para elaborar el diagnóstico y como se observa aquí, hacer una planeación estratégica con hoja de cálculo.

\section{Conclusiones}

La tecnología no tiene que ser abrumadoramente compleja o los tratamientos con excesiva sofisticación para ser válidos. La técnica que articula el diagnóstico institucional y la planeación estratégica utilizando un software comercial o uno de hoja de cálculo es de utilidad porque permite, paso a paso, valorar en una primera instancia el funcionamiento vital de la institución y, a partir de su diagnóstico interno, desarrollar las estrategias congruentes, pertinentes y necesarias para la mejora continua, interviniendo sobre las áreas de oportunidad y corrigiendo los focos rojos que constituyen las debilidades institucionales.

En el presente trabajo se procura explicar con claridad los resultados obtenidos y las posibilidades de mejora de la técnica utilizada, no obstante, se enfatiza que el estudio funciona con datos empíricos.

\section{Referencias}

Cano, J. A., \& Ayala, C. J. (2019). Metodología de enseñanza en cursos de logística para programas de administración de empresas. Formación universitaria, 12(2), 73-82

Chamizo, G. O. y M. P. Jiménez S. 1982. El análisis institucional. Perfiles Educativos (16). CISE-UNAM. México. http://www.iisue.unam.mx/perfiles/articulo/198 2-16-el-analisis-institucional.pdf

Gago Huguet, A., \& Mexico. Secretaría de Educación Pública. (2002). Apuntes acerca de la evaluación educativa. Retrieved from https://books.google.com.mx/books/about/Apu ntes_acerca_de_la_evaluación_educati.html?id =2RwnAQAACAAJ\&redir_esc =y
Instituto Politécnico Nacional. 2002. Metodología para el análisis FODA. Secretaría Técnica. Dirección de Planeación y Organización. p. 7. Marzo 2002. México.

Jorge Yarce AYER LA EDUCACIÓN, M. DE. (n.d.). INSTITUTO LATINOAMERICANO DE LIDERAZGO. Retrieved from http://liderazgo.org.co/images/documentos/201 0/macrotendencias_de_la_educacion.pdf

Muñoz López, T. (2005). La Evaluación Institucional, sus categorías de investigación. Primer Foro de Investigación Educativa "Realidades y Retos" CD Con Memorias in Extenso Formato Word, 13.

Muñoz López, T. (2009). Los Sistemas Educativos. La educación y las organizaciones que educan. Saltillo: Universidad Autónoma de Coahuila. ISBN: 978-968-9530-12-1.

Myrdal, G. (1978). Institutional Economics. Journal of Economic Issues, 12(4), 771-783. https://doi.org/10.1080/00213624.1978.115035 77

Orlich., J. M. (2008). El análisis FODA. Planeación Estratégica.

Ramírez, J. L. (2009). Procedimiento para La Elaboración de un Análisis FODA como una Herramienta de Planeación Estratégica en las Empresas. Ciencia Administrativa. https://doi.org/10.1093/mnrasl/slv177 